\title{
Experiencia de indagación sobre el uso y apropiación de tecnologías móviles en la escuela*
}

\author{
Research experience of use and ownership of mobile technologies in schools
}

Experiência inquérito sobre o uso e apropriação de tecnologias móveis escola

Recibido: diciembre de 2011

Ruth Molina Vásquez**

Aceptado: julio de 2012

\author{
Sergio Briceño Castañeda***
}

\section{Resumen}

Este artículo presenta una síntesis de la investigación realizada por el grupo Didactec, la cual fue financiada por el Ministerio de Educación Nacional. Gira en torno a la conformación de un Observatorio Nacional de Uso y Apropiación de Tecnologías Móviles en la Escuela. El proceso de investigación busca recopilar y analizar las experiencias realizadas en Colombia sobre el tema, para luego construir un plan estratégico dirigido hacia la puesta en marcha del Observatorio, junto con las experiencias analizadas en un micrositio web que, esperamos, haga parte del Portal de Colombiaaprende.

Palabras clave: tecnologías móviles, estrategias 1 a 1, implementación en procesos educativos

\begin{abstract}
This article presents a synthesis of research by the group DIDACTEC, with funding from the Ministerio de Educación Nacional, about the formation of a National Observatory for use and appropriation of Mobile Technologies in the School. The research process for collecting and analyzing experiences in Colombia on the subject, to the Strategic Plan for the implementation of the Observatory and visibility, along with the experiences analyzed in a microsite, which is expected to hold in the Portal Colombiaaprende.
\end{abstract}

Keywords: mobile technologies, strategies 1 a 1, implementation in educational processes

\section{Resumo}

Este estudo resume uma investigação da DIDACTEC grupo, com financiamento do Ministério da Educação, sobre a formação de um Observatório Nacional para o uso e apropriação das Tecnologias Móveis na Escola. O

Investigación realizada por el grupo Didactec, en torno a la conformación de un Observatorio Nacional de uso y apropiación de tecnologías móviles en la escuela. Contó con el financiamiento del Ministerio de Educación Nacional.

** Directora del grupo Didactec y Coordinadora de la Especialización en Educación en Tecnología de la Universidad Distrital Francisco José de Caldas. Correo electrónico: rmolinav@udistrital.edu.co

*** Investigador del grupo Didactec, docente de la Especialización en Educación en Tecnología de la Universidad Distrital Francisco José de Caldas y de la Licenciatura en Educación Básica con Énfasis en Tecnología e Informática de Uniminuto. Correo electrónico: sergiobri2012@gmail.com 
processo de pesquisa busca coletar e analisar as experiências da Colômbia sobre o assunto, com o Plano Estratégico para a implementação do Centro e torná-lo visível, junto com as experiências analisadas em um microsite, que se espera para a casa no Portal de Colombiaaprende .

Palavras-chave: as tecnologias móveis, estratégias de implementação 1 a 1 , em processos educativos

\section{Introducción}

Desde el año 2009, en el Programa de Nuevas Tecnologías del Ministerio de Educación Nacional, se expresó una gran preocupación por la expansión indiscriminada de experiencias educativas con uso de computadores portátiles en sectores públicos y privados. La preocupación radicaba en que dichas experiencias no estaban caracterizadas, ni sistematizadas, además, no se reconocía su estructura o el impacto en las instituciones educativas, ya que carecían de un mínimo control por parte del MEN.

Bajo este panorama, el Grupo de Investigación Didactec de la Universidad Distrital Francisco José de Caldas realizó un proyecto de investigación orientado a reconocer las características y el impacto de las experiencias adelantadas en el país con el uso de estas herramientas tecnológicas. El objetivo era implementar un Observatorio Nacional de Uso y Apropiación de Tecnologías Móviles en la Escuela, para generar un compendio de las características de estas tecnologías, analizar su uso e impacto y brindar orientaciones para el desarrollo de experiencias futuras o en marcha.

Este gran objetivo implica hacer un reconocimiento de todas las iniciativas que tienen lugar en el país, hacer una descripción de las mismas, determinar el tipo de tecnologías y sus usos, el soporte pedagógico de la experiencia, las estrategias de organización e investigación utilizadas y su impacto en la comunidad educativa. Una segunda instancia de la investigación, implica la creación de un espacio virtual que albergue y facilite la visualización y socialización de las experiencias pedagógicas que se llevan a cabo en el país y que involucran el uso de tecnologías móviles en la escuela; un espacio en el que se puedan consultar las orientaciones que el MEN considera se deben tener en cuenta en este tipo de experiencias.

El proyecto de investigación se realiza con una metodología de carácter documental, y está dividido en tres etapas diferentes: 1) el levantamiento de información de las experiencias de uso y apropiación de tecnologías móviles en la escuela, 2) el diseño de una página web del Observatorio Nacional de Uso y Apropiación de Tecnologías Móviles en la Escuela y 3) el desarrollo de actividades de orientación para este tipo de experiencias.

\section{Algunas experiencias previas}

En el contexto internacional se encuentran algunas experiencias que permiten comprender el papel de las tecnologías móviles en la escuela y que además brindan orientación sobre su proceso de incorporación pedagógica e investigativa.

En América Latina la preocupación por mejorar la calidad de la educación ha llevado a los gobiernos a generar procesos de inserción de tecnologías móviles en el aula de clase, como una apuesta para desarrollar experiencias eficaces, a través de la participación de fundaciones, ONG e instituciones de carácter privado (por lo general, proveedores de tecnología) y universidades. En la mayoría de estas experiencias se han desarrollado estrategias pedagógicas como el 1:1, que consiste en el uso de dispositivos móviles para actividades curriculares y extracurriculares que promueven cambios en la vida cotidiana de los estudiantes y sus familias. 
En países como Chile ${ }^{1}$, Venezuela ${ }^{2}$, Argentina ${ }^{3}$, México $^{4}$ y Uruguay $^{5}$ se han empleado de forma masiva computadores Classmate o $\mathrm{XO}$, como parte de una política estatal. El registro de estas experiencias se ha llevado a cabo en medios de comunicación que hacen énfasis en el número de computadores entregados, y mediante páginas institucionales que registran el día a día del desarrollo de estos proyectos. La implementación responde a un programa de carácter estatal, generalmente técnico, sobre el manejo de los dispositivos móviles, y de carácter pedagógico, frente al uso de estas herramientas para el trabajo de aula.

1 En Chile se encuentran dos proyectos: "Enlaces móvil 2.0: tecnología portátil en la sala de clases" y el programa "Wil@n", desde 2004 y 2008 respectivamente. Estos proyectos se fundamentan en estrategias 1@1, que ha beneficiado a 14.070 alumnos y docentes. Más información en: http://idbdocs.iadb.org/wsdocs/getdocument. aspx?docnum=849687 y http://www.mineduc.cl/index2. php?id_contenido=13359\&id_seccion $=10 \&$ id_portal $=1$

2 Canaima Educativo implementado desde el Ministerio de Educación y Deportes, es un plan rotativo con 300 alumnos de séptimo, octavo y noveno grado del Liceo Bolivariano Julio Bustamante de Caracas, a quienes se entregaron 40 computadoras tipo Classmate con un modelo de enseñanza 1:1. Para consultar más acerca de este proyecto, ingrese al siguiente enlace: http://www.canaimaeducativo. gob.ve/

3 Una laptop por chico" fue la propuesta del Ministerio de Educación de Argentina. Esta consistía en dotar a los alumnos de Netbooks conectados a Internet para ser utilizados en forma intensiva . Desde 2007, Educar viene desarrollando un proyecto piloto con 28 escuelas de la Argentina para experimentar el modelo con un millón de Netbooks. Para consultar mas información, ingrese a: http://www.educ.ar, y http://www.conectarigualdad.gob. ar/

4 El "Proyecto Edumóvil" en México busca mejorar el proceso de enseñanza y aprendizaje de los niños en primaria, a través de la incorporación de tecnología móvil en el aula (ver. http://www.utpl.edu.ec/ried/images/pdfs/eudomovil.pdf). El "Proyecto Mochila Digital", desarrollado por Telmex en las escuelas de primaria de Colima y Culiacan, es una iniciativa para la inserción de XO que hace énfasis en la exploración, expresión y construcción de conocimientos, con base en intereses personales, procesos de colaboración y aprendizaje significativo. Para ver más acerca de este proyecto consultar: http://fch.mxl.uabc.mx/ lateduca/141.pdf

5 El "Plan CEIBAL" es desarrollado entre el Ministerio de Educación y Cultura (MEC) de Uruguay, el Laboratorio Tecnológico del Uruguay (LATU), la Administración Nacional de Telecomunicaciones (ANTEL) y la Administración Nacional de Educación Pública (ANEP) desde el año 2007. Busca promover la inclusión digital, disminuyendo la brecha existente en la población entre quienes tienen y no acceso a los medios digitales. Más información en: http://www.ceibal.org.uy/
En la experiencia de Brasil ${ }^{6}$ y Costa Rica ${ }^{7}$ se plantea un desarrollo diferente, pues parten del desarrollo de experiencias piloto que prueban la eficiencia de los equipos y se preguntan por el diseño de estrategias pedagógicas para su implantación. También se reportan experiencias con el empleo de tecnologías móviles como PDA, celulares ${ }^{8}$, Pocket PC, etc., en su mayoría como ejercicios investigativos que buscan responder a las preguntas relacionadas con el contexto pedagógico o esperan servir de base para la formulación de proyectos y políticas educativas.

Así mismo, se realizan proyectos pilotos por parte del gobierno con apoyo de la empresa privada, como en Nigeria, Tailandia y en España, aunque con diferentes tipos de tecnología. En el caso de Tailandia ${ }^{9}$, se orienta a cubrir las necesidades de cobertura de la educación básica, con un modelo de 1:1, bajo la supervisión de la Universidad de Chulalongkorn y con el apoyo de Intel ${ }^{10}$, al igual que en Nigeria ${ }^{11}$, donde esta empresa (con aval de

6 Esta experiencia se desarrolla entre la Fundación Bradesco e Intel. El propósito es dotar a los alumnos de 40 escuelas con un computador portátil Para capacitar a los docentes se utiliza un curso básico de soporte técnico para estudiantes y el "Programa Entre Pares". Más información en: http://www.schoolchains.org/es/school/bradescofoundation-brazil

7 En Costa Rica se han realizado dos experiencias piloto. La primera, en la Escuela El Silencio, contó con el apoyo de la Fundación Omar Dengo. La Segunda se realizó en la Escuela Fidel Chaves con el apoyo de INTEL EDUCAR..

8 En el 2006 la Universidad Nacional de La Plata y la Universidad Tecnológica Nacional desarrollaron el proyecto "Integración de la tecnología móvil a los entornos virtuales de enseñanza y de aprendizaje. El propósito era implementar una estrategia de utilización del servicio de mensajes cortos de texto (SMS) a través de celulares, para realizar una actividad colaborativa de resolución de un problema.

9 Para dar solución al déficit de profesores y cumplir con la política educativa enfocada a la formación de ciudadanos en el marco de la globalización, Tailandia realiza una experiencia con Classmate generando nuevas dinámicas de enseñanza-aprendizaje, relacionadas con el manejo de computadores e Internet con el uso del modelo 1:1.

10 Intel ha apoyado varias experiencias piloto alrededor del mundo, las cuales se describen en: http://www.intel.com/ espanol/pressroom/releases/2007/0322.htm

11 Con el modelo de 1:1 proporcionado por Intel, la conexión a red mediante Wimax y capacitación a docentes en el uso de los equipos y el diseño de currículo, la experiencia de Nigeria se realizó en una zona en donde los (las) estudiantes nunca habían tenido contacto con un computador. Más información en http://www.intellearningseries. com/espanol/LearningSeries/SuccessStories.html 
la Agencia de Ciencia y Tecnología de ese país) realiza una experiencia piloto con 36 Classmate, en el distrito de Jabi.

En contrate, en España se realiza la experiencia de investigación Mobile Technologies for Ad-hoc Learning -MoTFAL ${ }^{12}$, por parte de la Universidad de Cádiz, que busca articularse en torno a Telecollaborative Curriculum Projects con el objeto de evaluar, valorar y analizar las posibilidades del uso de tecnología móvil (PDA, GPS) en los procesos de enseñanza y aprendizaje. El uso masivo de computadores portátiles se ha realizado en Estados Unidos en por lo menos tres experiencias: en el Estado de Maine, con una fase inicial (2002-2004) en la que se provee de portátiles a estudiantes y profesores de séptimo, octavo grado y a las escuelas de asistencia técnica y de desarrollo profesional para integrar la tecnología del portátil en el currículo $\mathrm{y}$ en las clases. Inspirados en el proyecto OLPC, el Distrito de Fullerton, California, busca que los estudiantes aprendan "cómo aprender", mediante una capacitación general del cuidado del portátil y del sistema operacional. Finalmente, el Estado de Texas dota a todos los estudiantes de las escuelas de computadores Apple, para fortalecer su desarrollo en las áreas en las que presentan mayores dificultades, aspecto que genera nuevos procesos de enseñanza-aprendizaje, mediante una estrategia pedagógica de 1:1.

\section{Algunos referentes teóricos}

De acuerdo con Vidart (1997), las técnicas, los procesos tecnógenos y sus productos encarnan todo un tecno sistema que podríamos denominar tecnosfera. La tecnología al interior de este tecnosistema inherente a las construcciones culturales se convierte en el cuerpo de estudio de las "tecne".

12 Esta experiencia busca potenciar el aprendizaje mediante el uso de tecnologías a las que los (las) estudiantes tienen un acceso cotidiano, en procesos de enseñanza y aprendizaje de escuelas de diferentes países, así como su utilización para la comunicación e intercambio de información entre varias escuelas. El proyecto concibe la enseñanza como una actividad compleja, simultánea, multidimensional, bajo tres principios: un proyecto educativo, la enseñanza para la comprensión y la telecooperación. Ver http://www.ea.gr/ep/motfal/common/arxeia/borrador_ spanish.pdf
Esta visión heurística permite ver la tecnosfera, limitando no sólo los procesos tecnológicos a la producción de materiales, sino las técnicas, como por ejemplo fisiotécnicas (propias de la inserción del cuerpo humano en la naturaleza), psicotécnicas (simbólicas, de previsión, explicación y comunicación al interior de todas las culturas) o demotécnicas (praxis al interior de las interacciones sociales, cánones de actuación inmersos en la cultura) (Vidart, 1997). Este tecnolosistema se compendia en un paradigma tecnológico, es decir, en un conjunto de conocimientos y técnicas que permiten un determinado dominio del medio natural y del desarrollo del sistema cultural en su conjunto, construido sobre una plataforma tecnológica diferente, que implica no solo el manejo de un puñado de instrumentos físicos, sino también la creatividad científica y los procesos de adaptación instrumental a cada cultura (Maya, 1998).

En términos de Javier Echeverria, las técnicas son entidades culturales de carácter abstracto, que tienen diferentes aplicaciones, se representan de diversas formas y se definen como el conjunto de realizaciones técnicas concretas posibles con una máquina. Se entiende una realización técnica como un sistema de acciones humanas intencionalmente orientado a la transformación de objetos concretos para conseguir de forma eficiente un resultado valioso, mientras que una realización tecnológica es un sistema de acciones humanas, industriales y de base científica, intencionalmente orientadas a la transformación de objetos concretos para conseguir estos resultados (Echeverría, 1996).

Teniendo en cuenta lo anterior y de acuerdo con Quintanilla (1989), la tecnología no ha de centrarse en los artefactos o en las máquinas, sino en las acciones que los seres humanos podemos llevar a cabo gracias a ellas. Esta opción filosófica tiene múltiples consecuencias, porque vincula la filosofía de la tecnología con la teoría de la acción, y minimiza así el papel de los instrumentos en las acciones técnicas y señala que los agentes de estas son las personas, no las máquinas; ello aleja toda forma de instrumentalismo y de determinismo tecnológico. Ahora, el uso de la gama de tecnologías, 
entre ellas las $\mathrm{TIC}^{13}$, implica un reconocimiento de sus posibilidades técnicas de incorporación, de los contextos de aplicación y de las intenciones de quien las utiliza. Las tecnologías móviles se convierten en un subsector de las TIC referido, tanto a los procesos de computación móvil, como de comunicaciones móviles y medios de emisión de datos, gracias a las ondas en el espectro radial, que suplen el problema de la conectividad entre los puntos móviles. Las primeras tecnologías móviles, beepers, redes móviles privadas, le dieron paso a las agendas personales, miniordenadores, laptops, y abrieron campo a las tecnologías móviles digitales (Eafit, 2009). Las tecnologías móviles permiten trasladar una actividad que generalmente ocurre en un espacio físico de lugar a otro, sin necesidad de estar en el lugar donde ocurre dicha acción. Es necesario reconocer que en las tecnologías móviles, más que equipos tecnológicos, se componen de sistemas y que como tal obedecen a los postulados de la teoría de sistemas con elementos que cumplen una función determinada y que pueden ser materiales (dispositivos portátiles, celulares, PDAs, tarjetas SIM, USIM y cualquier otro dispositivo que pueda emitir y recibir datos de manera inalámbrica), o no materiales (esquemas de conectividad como Wi-Fi, GPRS, Bluetooth, GSM, 2G y 3G, UMTS).

En los sistemas de tecnologías móviles, las relaciones que se establecen entre los dispositivos, los canales de comunicación y el software utilizado para el procesamiento de la información generan cambios en la velocidad, eficiencia, calidad y demás aspectos necesarios en la transmisión de datos, además de nuevas posibilidades derivadas de intercambiar dispositivos, canales y software asociado.

Las posibilidades descritas permiten la implementación de múltiples tecnologías móviles, lo cual genera múltiples tipos de estrategias pedagógicas para la incursión de las tecnologías móviles en la escuela, que es necesario analizar, socializar y visibilizar, como en este caso, mediante un observatorio,

13 En la tecnología se encuentran incluidas varias de sus manifestaciones, en las cuales las tecnologías de información y comunicación son solo una tecnología más, no la única. entendido este último como un sistema permanente y actualizado que busca integrar información parcialmente dispersa, aplicar estadísticas a la información con la idea de analizar y proyectar escenarios presentes y futuros, dirigido a ciudadanos en general, profesionales y público especializado. La información de los observatorios se convierte en un conocimiento útil con indicadores generales y específicos, sobre el estado en la gestión del conocimiento o en el diseño de políticas de uso y administración frente a lo que se observa. En el caso de las tecnologías móviles, un observatorio se convierte en el referente social que aporta el conocimiento necesario para dar respuesta a cualquier problema sobre estas, así como suministrar información a profundidad y completud sobre el desarrollo, la difusión, el uso de productos y servicios asociados (Gil, Rivero y Gil, 2003). En este caso en particular, tiene un fin mucho más específico en cuanto que asocia dos variables fundamentales, experiencias pedagógicas que utilizan intencionalmente tecnologías móviles en los espacios académicos.

En este sentido, una experiencia pedagógica se puede asumir como un saber que se construye en el proceso de enseñanza-aprendizaje, que se forma in situ, que aguza la mirada y evalúa los problemas que se van presentando en las prácticas educativas (Zuluaga, 2003). El saber, a diferencia del conocimiento, permite miradas menos universales, y se constituye como un punto de encuentro con otros saberes y disciplinas, integrándolos a posturas ideológicas, políticas, enriqueciendo discusiones intersubjetivas en la interacción de alumnos, maestros, comunidad educativa. Pero es el docente el que deviene este saber pedagógico en un saber diferente al cotidiano, integrado, reelaborado, repensado en y con el otro, y genera de esta manera transformaciones en el acto pedagógico. La experiencia pedagógica se asume como una dinámica particular de quienes viven y asumen este espacio, delimitado por la cultura, las relaciones y acciones; es una construcción simbólica intersubjetiva que tiene un significado, referentes y signos propios para la comunidad donde se desarrolla, y se asume como la realidad escolar derivada de dicha experiencia.

Tipificar las experiencias pedagógicas es arriesgado, especialmente si se tiene en cuenta que es 
una actividad de un grupo particular. Algunos autores aducen que el grado de desarrollo puede ser un factor fundamental, y que por tanto algunas de estas experiencias estarán en la fase inicial de propuesta de idea creativa, mientras que otras pueden estar ya consolidadas, validadas en más de un contexto; hecho que se constituye más en un conocimiento de carácter universal que en un saber.

Con respecto de las estrategias 1 a 1 , se encuentra que es una propuesta educativa que consiste en proveer a los alumnos de un curso de computadoras portátiles de bajo costo, preferentemente conectadas a Internet para utilizar de forma intensiva en el trabajo escolar. Personales y portables, estos dispositivos son especialmente indicados para el trabajo por proyectos, para la integración de diferente disciplinas, el trabajo en red y la exploración de la comunidad.

Los objetivos principales de esta estrategia están orientados a que las generaciones jóvenes adquieran destrezas y competencias basadas en las TIC, para que así se reduzca la brecha digital entre individuos y grupos sociales, y se mejoren las prácticas educativas y los logros académicos (Valiente, 2010, citado por Lagos, 2011). Lo anterior facilitaría el acceso, en la casa o en el colegio a partir de diversas actividades de aprendizaje, a software educativo, a la comunicación y colaboración entre compañeros y profesores, y a la inclusión de los padres en el aprendizaje de sus hijos.

\section{Metodología utilizada}

Con un enfoque de carácter cualitativo y diseño metodológico de investigación documental, el proyecto realiza un ejercicio de reconocimiento de elaboraciones conceptuales sobre la problemática, abordando preguntas asociadas a relaciones no establecidas, transformaciones generadas e influencias recibidas que pueden dar cuenta del rumbos conceptual del tema de estudio y de posibles horizontes de indagación (Morales, s.f.).

La investigación documental busca información, descubre el problema, establece conexiones, analiza, sintetiza e interpreta información para apropiarla y convertirla en conocimiento. Se conocen nuevos conceptos y se generan puntos de vista que favorecen la compresión y generación de significados, de nuevas elaboraciones y/o reflexiones.

La interpretación se realiza mediante técnicas, reglas y procedimientos de acumulación del conocimiento; compilación de evidencias sobre el estado de las experiencias indagadas; recomprensión del objeto de estudio ${ }^{14}$; reflexión crítica para llegar a nuevas explicaciones, y reconstrucción del objeto de estudio. Así pues, las actividades del proyecto en cada una de las etapas de desarrollo son:

- Levantamiento de información: búsqueda y recopilación de experiencias nacionales, elaboración de categorías de descripción de las experiencias, selección y clasificación de la información en las categorías generadas, identificación y comunicación con contactos de cada una de las experiencias, recopilación de información documental y realización de entrevistas con los contactos, elaboración de una matriz de resumen y Análisis preliminar a la luz de las categorías planteadas.

- Diseño página del Observatorio Nacional de Uso y Apropiación de Tecnologías Móviles en la Escuela: elaboración del formato pedagógico conceptual de la página, diseño de la arquitectura de la página e interfaz y desarrollo de la página.

- Realización de las orientaciones del Observatorio Nacional de Uso y Apropiación de Tecnologías Móviles en la Escuela: horizonte institucional, diseño de la estrategia metodológica y realización del plan de acción.

Se plantean ocho categorías de análisis ${ }^{15}$ con las cuales se busca clasificar la información indagada. Cada una de ellas tiene subcategorías para caracterizar aspectos más concretos y descriptores que detallan elementos constitutivos de cada experiencia.

14 Se realiza con el objeto de descubrir nuevas facetas, problemas o conclusiones del tema objeto de investigación.

15 Estas categorías han sido revisadas en relación con los elementos propuestos por el MEN, la construcción de metadatos y con los requerimientos que van surgiendo en la elaboración de la propuesta del Observatorio Nacional de Uso y Apropiación de Tecnologías móviles en la escuela. 


\begin{tabular}{|c|c|c|}
\hline \multicolumn{2}{|l|}{ Categoría y subcategorías } & Descriptor \\
\hline \multirow{9}{*}{\multicolumn{2}{|c|}{ INFORMACIÓN GENERAL }} & Nombre del proyecto \\
\hline & & Institución patrocinadora \\
\hline & & Entidad ejecutora \\
\hline & & Fecha de inicio \\
\hline & & Fecha de culminación \\
\hline & & Departamento \\
\hline & & Municipio \\
\hline & & Localidad \\
\hline & & Cobertura (institucional, local, departamental, regional, nacional) \\
\hline \multirow{14}{*}{\multicolumn{2}{|c|}{ INSTITUCIONES PARTICIPANTES }} & Nombre de las instituciones \\
\hline & & Sede \\
\hline & & Tipo (institución educativa, centro educativo, otro) \\
\hline & & Sector de aplicación \\
\hline & & Localización (rural o urbano) \\
\hline & & $\begin{array}{l}\text { Contexto de aprendizaje: (primera infancia, preescolar, básica pri- } \\
\text { maria, secundaria, media, media técnica/pedagógica, tecnológica, } \\
\text { técnica profesional, universitario, posgrado, no formal, gestión } \\
\text { administrativa, territorial, extensión, bienestar, formación laboral) }\end{array}$ \\
\hline & & Grado de escolaridad \\
\hline & & $\begin{array}{l}\text { Tipo de participante (estudiantes, docentes, familia y comunidad, } \\
\text { directivos, investigadores, otras) }\end{array}$ \\
\hline & & Número de estudiantes beneficiados \\
\hline & & Número de docentes involucrados \\
\hline & & Número de familias participantes \\
\hline & & Número de directivos \\
\hline & & Número de investigadores \\
\hline & & Otros participantes \\
\hline \multirow{14}{*}{ TIPO DE TECNOLOGÍA } & \multirow{4}{*}{ Dispositivos } & Tecnología móvil utilizada \\
\hline & & Características \\
\hline & & Cantidad \\
\hline & & Periféricos \\
\hline & \multirow{2}{*}{ Conectividad } & Tipo (inalámbrica, alámbrica, wi-fi, bluetooth, infrarrojo, ppt, otro) \\
\hline & & Características \\
\hline & \multirow{8}{*}{ Contenidos } & Herramientas de productividad utilizadas \\
\hline & & Contenidos desarrollados para procesos de enseñanza - aprendizaje \\
\hline & & OVAS \\
\hline & & Otros contenidos \\
\hline & & Herramientas de comunicación utilizadas \\
\hline & & Herramientas colaborativas utilizadas \\
\hline & & Sistema operativo \\
\hline & & Tipo de contenidos utilizados \\
\hline
\end{tabular}




\begin{tabular}{|c|c|c|}
\hline \multicolumn{2}{|l|}{ Categoría y subcategorías } & \multirow{2}{*}{$\begin{array}{l}\text { Descriptor } \\
\text { Enfoque utilizado }\end{array}$} \\
\hline \multirow{12}{*}{ COMPONENTE PEDAGÓGICO } & \multirow{6}{*}{$\begin{array}{l}\text { Propuesta } \\
\text { Curricular }\end{array}$} & \\
\hline & & Tipo de Metodología \\
\hline & & Estrategias de rediseño curricular \\
\hline & & Tipo de proyecto (área, aula, institucional, combinado, no definido) \\
\hline & & Evaluación de la estrategia \\
\hline & & Áreas de implementación \\
\hline & \multirow{6}{*}{ Formación } & Participantes (estudiantes, docentes, directivos, familias, otros) \\
\hline & & Modalidad \\
\hline & & Formación de Docentes (de carácter axiológico, cognitivo y social) \\
\hline & & Directivos (Elementos de carácter axiológico, cognitivo y social) \\
\hline & & Estudiantes (Elementos de carácter axiológico, cognitivo y social) \\
\hline & & Familias (Elementos de carácter axiológico, cognitivo y social) \\
\hline \multirow{11}{*}{ SOPORTE TECNOLÓGICO } & \multirow{2}{*}{ Dispositivos } & Mantenimiento preventivo \\
\hline & & Mantenimiento correctivo \\
\hline & \multirow{4}{*}{$\begin{array}{l}\text { Estrategia de } \\
\text { soporte }\end{array}$} & Equipo de apoyo general \\
\hline & & Equipo de apoyo en campo \\
\hline & & Mesa de ayuda \\
\hline & & Implementación de la estrategia \\
\hline & \multirow{3}{*}{ Contenidos } & Antivirus \\
\hline & & Mantenimiento preventivo de contenidos \\
\hline & & Restricciones (filtros y controles) \\
\hline & \multirow[b]{2}{*}{ Conectividad } & Mantenimiento preventivo (router, acces point, servidores) \\
\hline & & $\begin{array}{l}\text { Mantenimiento correctivo de equipos (router, acces point, } \\
\text { servidores) }\end{array}$ \\
\hline \multirow{16}{*}{ ESTRATEGIA INVESTIGATIVA } & \multirow{7}{*}{$\begin{array}{l}\text { Entidades } \\
\text { acompañantes }\end{array}$} & Problema abordado \\
\hline & & Metodología utilizada \\
\hline & & Categorías de análisis \\
\hline & & Resultados \\
\hline & & Conclusiones \\
\hline & & Autoevaluación \\
\hline & & Estrategia de socialización \\
\hline & \multirow{9}{*}{ Institución } & Intención de investigación \\
\hline & & Autoevaluación \\
\hline & & Etapas del proceso \\
\hline & & Resultados \\
\hline & & Conformación del equipo \\
\hline & & Área y nivel de formación \\
\hline & & Etapas de desarrollo del proyecto \\
\hline & & Tipo de proyecto (aula, área, transversal, etc.) \\
\hline & & Documentos informativos \\
\hline
\end{tabular}




\begin{tabular}{|c|c|c|}
\hline \multicolumn{2}{|l|}{ Categoría y subcategorías } & Descriptor \\
\hline \multirow{8}{*}{ ESTRATEGIA ORGANIZATIVA } & \multirow{5}{*}{$\begin{array}{l}\text { Equipo de } \\
\text { Acompañamiento }\end{array}$} & Conformación del equipo \\
\hline & & Área y nivel de formación \\
\hline & & $\begin{array}{l}\text { Etapas de desarrollo del acompañamiento realizado a los docentes } \\
\text { y las instituciones }\end{array}$ \\
\hline & & Documentos informativos \\
\hline & & Socialización del proyecto \\
\hline & \multirow{3}{*}{ Institución } & $\begin{array}{l}\text { Logística institucional (modificaciones en el horario escolar, tiem- } \\
\text { pos destinados para las formaciones, en el tamaño de grupos, otros) }\end{array}$ \\
\hline & & $\begin{array}{l}\text { Planta Docente (cambios en planta docente, en horarios y carga } \\
\text { académica) }\end{array}$ \\
\hline & & $\begin{array}{l}\text { Planta Física (Adecuación de salones y la planta física de la } \\
\text { institución) }\end{array}$ \\
\hline \multirow{4}{*}{\multicolumn{2}{|c|}{ IMPACTO DE LA EXPERIENCIA }} & En la institución \\
\hline & & En los estudiantes \\
\hline & & En la comunidad \\
\hline & & En el docente \\
\hline
\end{tabular}

Tabla1. Categorías de análisis de las experiencias.

Fuente: elaboración propia

\section{Resultados obtenidos}

\section{Desde las experiencias indagadas}

De un total de 81 experiencias que incorporan tecnologías móviles en el ámbito escolar, se documentaron $45^{16}$, la mayoría financiadas por el sector público, ejecutadas en instituciones de carácter público y en marcha. El ejercicio descriptivo de las experiencias y su análisis se realiza a partir de la información obtenida en páginas web, documentos, informes de avance o finales, encuestas en línea, entrevistas a los directores y visitas a las instituciones participantes. Esta información se somete a un proceso de triangulación que permite complementar los datos de cada una de las iniciativas. Se observa que algunas experiencias tratan de mantenerse de acuerdo con los procesos de las instituciones que realizan la inclusión de proyectos relacionados con tecnologías móviles; sin embargo,

16 El criterio de inclusión de las experiencias de incorporación de tecnologías móviles en procesos educativos encontradas a nivel nacional se fundamenta en el acceso a la información facilitada por las experiencias mismas, a nivel documental, de entrevistas y visitas, en el momento del análisis. en algunas no es evidente el desarrollo y la apropiación del discurso de sus componentes.

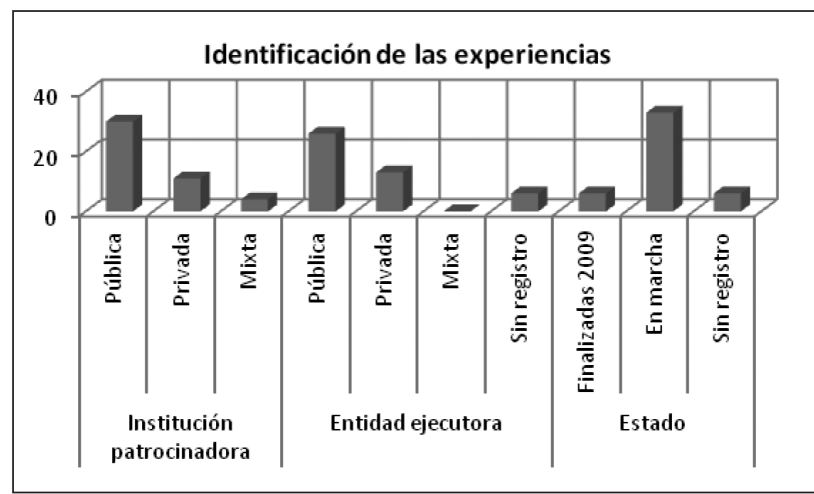

Gráfica 1. Identificación general de las experiencias documentadas.

Fuente: elaboración propia

En otras experiencias, las propuestas pedagógicas incursionan dentro de un modelo participativo e incluyente, enmarcado en un enfoque constructivista, preocupado por el aprendizaje colaborativo y autónomo, así como por el desarrollo de competencias y valores. En estas experiencias se observa la participación de docentes y estudiantes, que son los actores que se encuentran más activos dentro 
de las instituciones; sin embargo, no es ajeno el hecho de que un número reducido de experiencias han incluido la participación de padres de familia en espacios de interacción y formación. En el componente tecnológico se encuentra que la mayoría de las experiencias que se relacionan con el uso de portátiles, ya sean de tipo convencional o tipo Classmate con sistema operativo Windows, o XO de OLPC que emplean el sistema Linux, cuentan con acceso a Internet por medio de conectividad inalámbrica. Adicionalmente, es evidente la existencia parcial de equipos de acompañamiento técnico en algunas experiencias, mientras que en la mayoría son inexistentes.

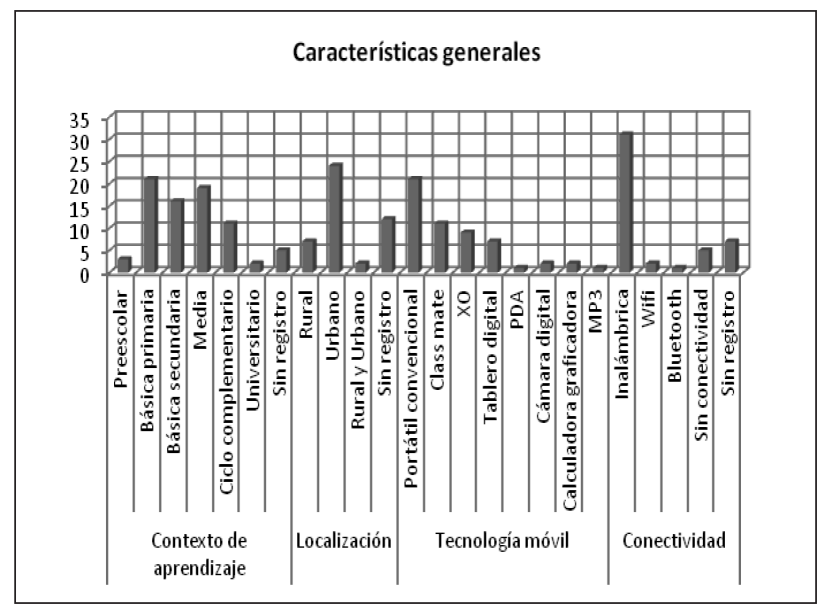

Gráfica 2. Características generales de las experiencias.

Fuente: elaboración propia

La implementación de tecnologías móviles se orienta al uso de computadores portátiles, aunque se encuentran experiencias que utilizan tableros digitales, PDAs (Pocket PC) y calculadoras; aspecto que indica la necesidad de continuar indagando en la concepción de los artefactos que hacen parte de las tecnologías móviles y su inclusión en el aula de clase. Se evidencia el uso indiscriminado de términos que parecen significar lo mismo en contextos diversos, como "aula digital", "aula inteligente", "aula virtual", "aula móvil", o sin hacer claridad conceptual de términos como tecnología o tecnología móvil, lo que amerita realizar algún tipo de aclaración.
El impacto de las experiencias se orienta a la motivación de docentes y estudiantes hacia el uso de las tecnologías en los procesos educativos. En ese sentido, la mayoría de experiencias adelantadas muestran la intención de realizar capacitación a docentes, en razón a la necesidad de cualificar la manera como estos abordan la implementación de dichas tecnologías en sus propuestas pedagógicas y actividades didácticas.

En algunas experiencias existe preocupación por iniciar procesos de pilotaje, para apoyar el desarrollo de áreas específicas del currículo, como una forma de hacer acercamiento a las dinámicas de trabajo pedagógico. Cabe resaltar que algunas iniciativas se han dado de manera autónoma por parte docentes motivados e interesados en generar espacios de aprendizaje, tanto para sus estudiantes, como para sí mismos y la comunidad escolar.

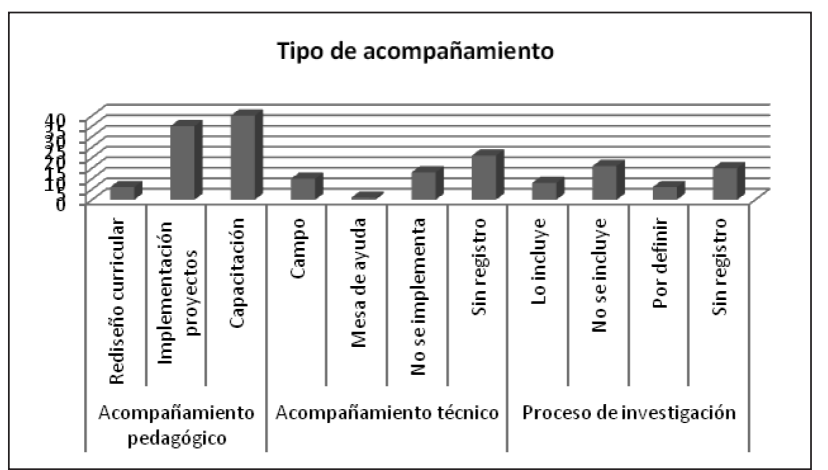

Gráfica 3. Tipo de acompañamiento en las experiencias.

Fuente: elaboración propia

\section{Desde el Observatorio Nacional de Uso y Apropiación de Tecnologías Móviles en la Escuela}

Con base en los anteriores resultados, se considera que la misión del Observatorio Nacional de Uso y Apropiación de Tecnologías Móviles en la Escuela es visibilizar, orientar y acompañar las iniciativas adelantadas en Colombia, en el uso y apropiación de tecnologías móviles en la escuela. Se espera, además, que se consolide como un referente para el diseño de políticas nacionales que orienten la puesta en marcha de experiencias en este sentido, a partir de su interacción, socialización y valoración. 
Los objetivos generales se encaminan a integrar información acerca de las tecnologías móviles en la escuela (parcialmente dispersa), con la idea de analizar y proyectar escenarios presentes y futuros de implementación, y generar conocimiento en torno a la definición de políticas nacionales que orienten el uso pedagógico, técnico, investigativo y la apropiación de este tipo de tecnologías.

A continuación se plantean las siguientes áreas temáticas para el trabajo transversal del Observatorio:

- Experiencias de uso y apropiación de tecnologías móviles: rastreo, descripción, documentación, sistematización, socialización y valoración de experiencias pedagógicas que se apoyan en el uso y apropiación de tecnologías móviles.

- Avances en tecnologías móviles: reconocimiento de ventajas y posibilidades de las tecnologías móviles; difusión de los avances alcanzados por parte de la industria tecnológica y del manejo de los dispositivos, sistemas y plataformas de última generación.

- Investigación e innovación pedagógica: la generación de conocimiento en torno al impacto del uso de tecnologías móviles en el ámbito escolar, los enfoques pedagógicos más acertados en la implementación de las mismas y los resultados de las experiencias representan algunos de los ámbitos a nivel investigativo.

- Políticas Nacionales: aportes y orientaciones que el Ministerio de Educación Nacional realiza con miras a cualificar las experiencias en torno al uso y apropiación más acertados de estas tecnologías móviles en los procesos educativos.

El Observatorio se desarrolla mediante tres fases secuenciales que buscan avanzar en la indagación, socialización de experiencias y construcción de políticas por parten del Ministerio de Educación Nacional, que orienten los planteamientos teóricos y las prácticas pedagógicas. Cada fase plantea un plan de acción que incluye objetivos, actividades, perfiles de ejecución, tiempos y resultados esperados. Estas fases son:
- Fase 1 - Conformación del observatorio: busca establecer las condiciones, criterios, planes de acción para su funcionamiento, los ejes y campos de trabajo por desarrollar. Es una fase de planeación, de organización y de configuración del Observatorio.

- Fase 2 - Consolidación del observatorio: busca visibilizar las experiencias en el área, socializar la información contenida en la página y generar acciones tendientes a caracterizar y sistematizar las experiencias que se vienen realizando en el país, además de estimular y acompañar nuevas iniciativas que busquen cualificar los procesos de enseñanza-aprendizaje en el contexto escolar, apoyados con tecnologías móviles.

- Fase 3 - Expansión del Observatorio: busca proponer criterios cualificados de valoración de experiencias, que permitan determinar las mejores prácticas pedagógicas, las experiencias significativas que se realizan en el país con el uso de los diferentes tipos de tecnologías móviles y comparar su impacto en el contexto educativo.

\section{Desde el micrositio del Observatorio}

Como parte de la estrategia de realización de las orientaciones para el Observatorio Nacional de Uso y Apropiación de Tecnologías Móviles en la Escuela, se diseña y desarrolla un micrositio web para acceder desde el portal Colombia Aprende, en tres fases: inicial, de análisis y de diseño.

En la fase inicial se realiza la gestión del micrositio $^{17}$ a partir de criterios de responsabilidad y cronograma de actividades. En la fase de análisis se desarrolla el documento conceptual del micrositio, incluyendo sus objetivos, población objeto y propuesta de diseño de interfase; adicionalmente, se describen las estrategias de divulgación, los procesos de actualización de información y una

17 El proceso de diseño y desarrollo del micrositio, en términos de fundamentación, plataformas utilizadas y niveles de programación, responde a las disposiciones solicitadas por el equipo técnico del Portal Colombiaaprende, en el cual se enlaza con el visto aprobatorio del Ministerio de Educación Nacional. 
descripción narrativa del micrositio. En la fase de diseño se expone la arquitectura de la información y el mapa de navegación del micrositio, el diseño educativo y pedagógico, y el diseño funcional final.

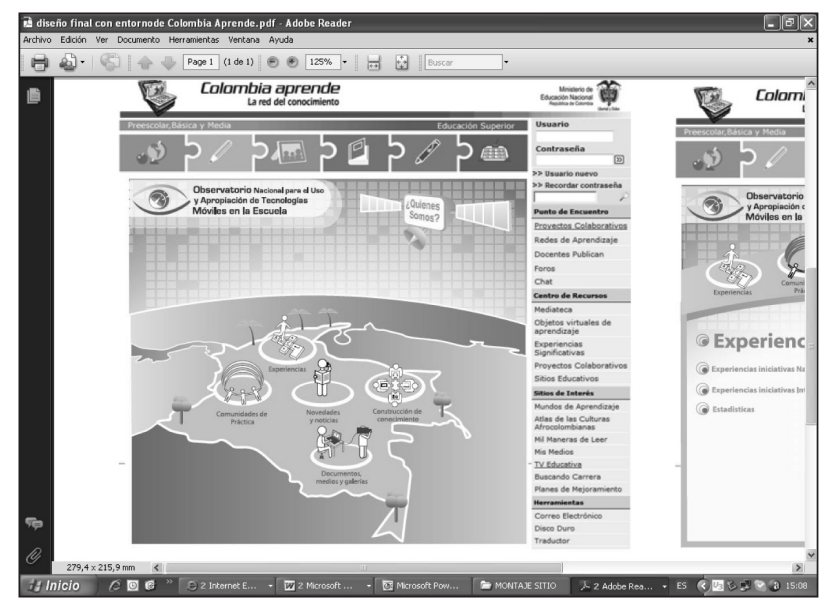

Gráfica 4. Pantalla inicial del micrositio del Observatorio Nacional de Uso y Apropiación de Tecnologías Móviles en la Escuela.

Fuente: elaboración propia

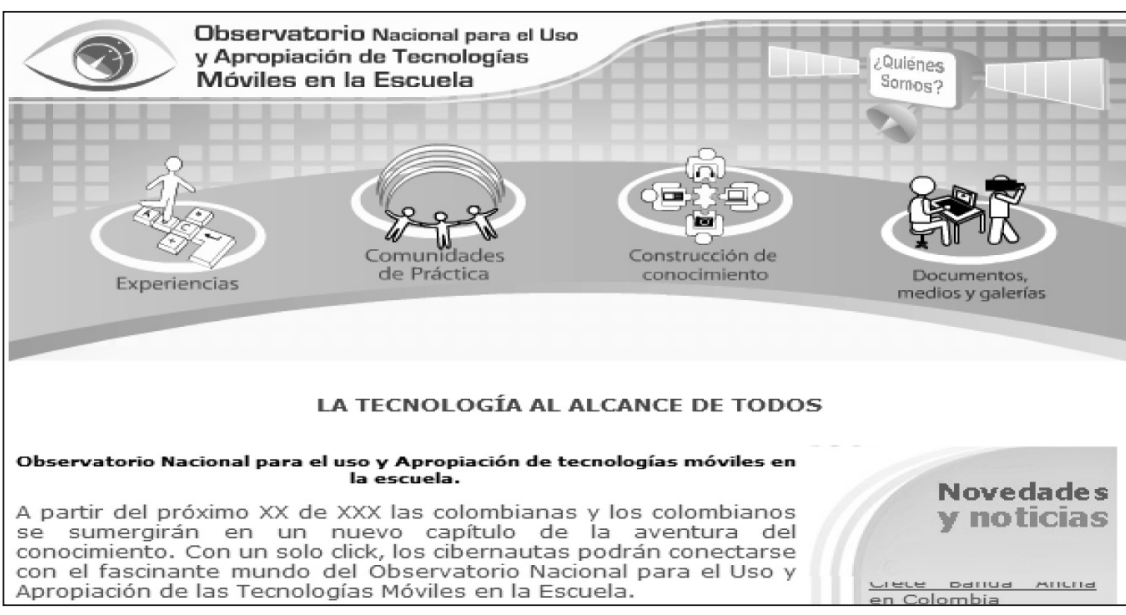

Gráfica 5. Diseño pantalla de presentación de información.

Fuente: elaboración propia

\section{Reflexiones y convergencias desde la diversidad}

Las reflexiones que se generan a partir de la interpretación de los resultados expuestos se presentan no con el ánimo de brindar respuestas concluyentes, sino de recoger elementos que puedan constituirse en referentes para ser problematizados y/o analizados en las dinámicas de trabajo del Observatorio
Nacional de Uso y Apropiación de Tecnologías Móviles en la Escuela. Se, además, presentan a partir de preguntas que abonan el terreno sobre la diversidad de matices que alimentan la discusión.

\section{Tecnologías móviles, ¿para qué?}

Cuando se pregunta acerca de las motivaciones que hacen posible la incorporación de tecnologías y su pertinencia, se encuentra un alto porcentaje de patrocinios provenientes del sector público como ministerios, gobernaciones y alcaldías, lo cual puede asociarse a intereses relacionados con políticas públicas o calidad de la educación. Es posible que las instituciones educativas públicas ante retos como calidad, cobertura y responsabilidad en la cualificación del capital humano del país deseen emprender acciones de inclusión de tecnologías en sus dinámicas pedagógicas. Si bien se percibe un gran interés de las instituciones, es inquietante ver cómo la inclusión de tecnologías se enfrenta con contextos inmersos en problemáticas diversas y con necesidades educativas particulares. En ese sentido, se abre un terreno amplio de discusión que abarca aspectos no solo de orden educativo, sino también económico, político y social, en los cuales la incursión de la tecnología responde más a presiones del mercado, que a las necesidades educativas y a los modelos de integración de las mismas en las prácticas educativas.

\section{¿Solos o acompañados?}

A pesar de que se identifica fácilmente el patrocinio del Estado a las experiencias, se observa un acompañamiento pedagógico centrado en la capacitación técnica y uno técnico casi inexistente. Esto genera inquietud sobre el papel de las entidades patrocinadoras, que desatienden de alguna manera procesos relevantes los cuales se desprenden del acompañamiento a las experiencias. Lo anterior lleva a pensar en la necesidad -si bien no urgente, sí central- de fortalecer 
aquellos procesos adelantados en las instituciones que de una manera $u$ otra requieren de una retroalimentación permanente y de un trabajo importante de seguimiento y evaluación por parte de entidades con experiencia en procesos de similar envergadura.

Esta reflexión remite necesariamente a la pregunta acerca de cómo se entienden los procesos de acompañamiento a las experiencias y sobre los planes y proyectos que en esta dirección se conciben en momentos previos a dotar a las instituciones de equipos $^{18}$, con perspectivas de acompañamiento a corto, mediano y largo plazo. Cabe mencionar que la existencia de experiencias que parten de iniciativas institucionales propias requiere también de un espacio desde donde se dinamice un acompañamiento asertivo, quizás mediado por grupos de discusión que posibiliten el intercambio constante, el trabajo en red y que brinden asesoría en diversos aspectos.

\section{¿Y por qué el turno de las tecnologías?}

Un alto porcentaje de las experiencias analizadas se genera a partir del año inmediatamente anterior y del año en curso, y de esta manera se aúna quizás al panorama de consolidación de ciertas políticas públicas desde las que se desprenden el tema de los patrocinios, la preocupación por la calidad y la cobertura, entre otros; pero también a las dinámicas mundiales de desarrollo, que perfilan trabajos interesantes de inclusión de tecnologías en los procesos educativos.

Los entornos urbanos siguen siendo los espacios donde mayoritariamente se vienen adelantando este tipo de experiencias con tecnologías móviles, en algunos casos bajo el pretexto de una gran demanda social, aunque en ocasiones llegan sin una solicitud expresa de las instituciones, sin planteamientos claros de inclusión o relaciones efectivas con su trabajo previo. La función de la escuela en las nuevas dinámicas tecnológicas y sociales es un tema complejo de abordar, aunque lo encontrado

18 Haciendo aquí énfasis en las experiencias con tecnologías móviles como computadores de diferente clase, puesto que la gran mayoría de experiencias son desarrolladas con este tipo de tecnologías. en las experiencias rastreadas muestra relaciones de las dinámicas internas de organización pedagógica para enfrentar los retos de apropiación de tecnologías y las acciones que se emprendan en esa dirección. Por ello, se percibe en varias experiencias un problema de coherencia entre sus enfoques y tendencias pedagógicas, con las metodologías de trabajo desarrolladas en torno al tema de las tecnologías.

Se vislumbra la necesidad de dejar de lado perspectivas meramente instruccionales y pasar a miradas más complejas de las relaciones que en términos de roles, acciones y procesos se construyen en el trabajo con tecnologías, aunado a los reportes sobre cambios en las dinámicas curriculares, que se constituyen en un paso hacia procesos de autonomía y de problematización de la estructura escolar enfrentada al reto de inclusión de tecnologías. Si bien estos reportes no son en su mayoría claros, dado que muchas experiencias aún se encuentran en proceso de implementación, el hecho de encontrar alusiones a necesidades detectadas con respecto al cambio de dinámicas internas de organización, no solo operativa, sino también pedagógica, permite pensar en la importancia de incluir en los procesos de acompañamiento y formación, cambios coherentes con las acciones pedagógicas.

\section{Tecnologías y/o comunidad educativa ¿Quiénes son los protagonistas?}

Se encuentran dos agentes relevantes en las dinámicas escolares de inclusión de tecnología. Por un lado, están las tecnologías móviles utilizadas; por otro, los procesos adelantados con la comunidad educativa para llevar a cabo esa inclusión tecnológica, y los cuales corresponden tanto a las visiones de escuela, como a enfoques y tendencias pedagógicas que orientan el trabajo institucional. Un porcentaje significativo de experiencias adelanta su trabajo con computadores portátiles de diversa clase, hecho que orienta la reflexión sobre la incorporación de otras tecnologías móviles, sobre todo de los maestros en quienes se concentra la mayor cantidad de acciones de formación en torno al trabajo con tecnologías. Valdría la pena preguntarse qué tan preparados se encuentran los maestros para 
adelantar experiencias con tecnologías diferentes al computador y cómo avanzar en la interacción de estos con otras tecnologías, cuando los procesos de cualificación se enfocan a procedimientos instrumentales de uso de los computadores

Por otra parte, llama la atención que no se utilicen de forma más difundida herramientas de comunicación y colaboración, cuando este es uno de los objetivos principales del uso de tecnologías móviles. Esto puede deberse a que se parte de una idea de formación inicial de corte instrumental, orientado a una inclusión progresiva a trabajos con herramientas de otro tipo. Es necesario entonces propender por el fortalecimiento del trabajo con herramientas colaborativas, de comunicación, software libre y educativo, que permitan reconocer la interdisciplinariedad en las dinámicas de trabajo escolar, puesto que si bien algunas experiencias consideran importante el trabajo con tecnologías en todas las áreas, sigue siendo constante la tendencia a abordar dicho trabajo desde las llamadas áreas básicas.

En ese orden de ideas, también es interesante observar que las iniciativas se concentran en mayor proporción en grados de educación secundaria y media. Esto puede deberse a varios factores, como el interés de formación de competencias laborales y las facilidades de hacer aplicaciones en el aula con estudiantes en una edad y/o etapa de desarrollo que le permiten desempeñarse en grupos de trabajo colaborativo, trabajo autónomo, participativo y de alto nivel propositivo. Es necesario hacer inclusión de las familias, en las cuales se piensa en términos más informativos que formativos, para otorgarles un papel central en el desarrollo de estrategias 1:1, al igual que de los directivos como actores importantes en los aspectos de organización y administración de las instituciones escolares.

\section{¿Dónde quedan los procesos de investigación?}

El rol de la investigación en estos procesos, si bien puede considerarse como relevante debido a las posibilidades de transformación y compresión de la propia práctica que esta ofrece, es un factor poco dimensionado en los procesos de inclusión de tecnologías; aspecto que hace necesario el emprendimiento de acciones de cualificación en este aspecto y de reflexión sobre los porqué y para qué de las tecnologías móviles en las prácticas pedagógicas. Es interesante mencionar cómo la gran mayoría de experiencias que, si bien se consideran a sí mismas exitosas, no contemplan aspectos referidos a procesos de investigación; situación que pone en evidencia una acción mediada por intenciones que muchas veces se quedan sin ser sistematizadas como evidencia de un proceso y como referente de transformación, calidad e impacto positivo. Este desinterés o dificultad para configurar procesos en ese sentido deja sin argumentos el florecimiento de otras experiencias, pues no se reconocen de forma sistematizada y socializada los resultados efectivos de los procesos realizados.

\section{Impactos, ¿hacia dónde se proyectan las trans- formaciones?}

Las experiencias se quedan cortas en la manera como dimensionan sus impactos, puesto que pocas presentan transformaciones en aspectos relacionados con roles de docentes, de estudiantes, en los procesos de enseñanza y aprendizaje, así como en asuntos relacionados con propuestas curriculares y metodológicas. Esto puede deberse a que muchas de ellas están aún en proceso de implementación y seguramente luego de un proceso juicioso de evaluación podrán dar cuenta de sus diversos niveles de incidencia. Aún así es importante no perder de vista este aspecto, ya que los procesos de evaluación permiten no solo reconocer la experiencia en sí misma, sino promover su transformación, y así contrastar los hechos con lo logrado y lo deseado. Esto conduce hacia una escuela diversa e inclusiva que ve en las tecnologías un medio para aportarle a la formación de seres humanos capaces de afrontar los nuevos retos sociales con una mirada crítica de su papel como agente de transformación.

\section{Visibilización y políticas, ¿aportan al estado de las prácticas pedagógicas?}

En Colombia es necesario aprender de las experiencias desarrolladas, ubicar los obstáculos y potencializar los aspectos que impactan positivamente 
al sector educativo que utiliza tecnologías móviles. Solo de esta manera es posible desarrollar acciones intencionales que redunden en una mejor calidad de vida de los colombianos, contribuyan a disminuir la brecha digital y a desarrollar habilidades que nos permitan como sociedad enfrentarnos a las exigencias del mundo actual. Por ello es absolutamente necesario no solo que se sistematicen y visibilicen estas experiencias, sino que se generen lineamientos que orienten la puesta en marcha de un sin número de iniciativas que puedan capitalizar los aprendizajes logrados y así aseguren procesos educativos más cualificados para todos los niños y jóvenes colombianos.

\section{Bibliografía}

Balardini, S. A. (s.f.). Jóvenes e identidad en el ciberespacio. Recuperado en mayo de 2007, de www.proyectojuventud.com.ar/tics/JOVENES_en_el_ciberespacio.doc

De Zubiría, J. (2006). Hacia una Pedagogía Dialogante. Bogotá: Editorial Magisterio.

Echeverría, J. (1996). Tecnociencia e interacciones a distancia. En M. Cruz (coord.), Acción humana, pp. 164-183. Barcelona: Ariel.

Gil, A., Feliu, J., Rivero, I. y Gil, E.P. (2003). ¿Nuevas tecnologías de la información y la comunicación o nuevas tecnologías de relación? Niños, jóvenes y cultura digital. Recuperado el 28 de mayo, de http://www.uoc.edu/dt/20347/index.html

Gross, B. (s,f.). El aprendizaje colaborativo a través de la red: límites y posibilidades. Recuperado en mayo de 2007, de http://www.uninorte.edu. co/congresog10/conf/08_El_Aprendizaje_ Colaborativo_a_traves_de_la_red.pdf

Johnson, D.H y Jonson, F.P. (2000). Joining Together. Minnesota: Minnesota University, Allyn and Bacon.

Maya, A. (1998). El retorno a la Tierra: introducción a un método de interpretación ambiental. Cuadernos ambientales, núm. 3.

Quintanilla, M.A., (1989). Tecnología: un enfoque filosófico. Madrid: Fundesco,
Gil G. y Gaitán P. (2004). Observatorios de Accesibilidad en Nuevas Tecnologías. Recuperado en febrero de 2009, de http:// www.tecnoneet.org/docs/2004/2-32004.pdf

Lagos, M.E. y Silva, J. (2011). Estado de las experiencias 1:1 en Iberoamérica. Revista Iberoamericana de Educación,56. pp. 75-94.

Ministerio De Educación Nacional (2008). Orientaciones generales para la educación en tecnología. Bogotá.

Perez, I. y Sacristan, J. (2004). Comprender y transformar la enseñanza. Madrid: Ediciones Morata.

Regil, L. (2002). Estrategias docentes para educar con nuevas tecnologías de la comunicación. Segundo Congreso de Imagen y Pedagogía. Mazatlán, Sinaloa.

Regil, L. (s.f.). La construcción de la mirada. Recuperado el 20 de febrero de 2007, de http:// www.hipertexto.info/documentos/\#Regil

Rizo, M. (2005). Me comunico, luego existo. El papel de la comunicación en la construcción de identidades. En: Culturales. Universidad Autónoma de Baja California. V.1, N.001. En: http://redalyc.uaemex.mx/redalyc/pdf/694/69410106. pdf Consulta: Febrero de 2009

Rojas, J. Olaya, J. y Rozo, C. (s.f.). Análisis de la Cultura TIC en la UPN. Recuperado en febrero de 2009, de http://www.unige.ch/fapse/clidi/ textos/CulturaTicUpn_Corzo_Olaya_Rojas.pdf

Vidart, D. (1986). Filosofía ambiental: el ambiente como sistema. Colombia: Editorial Nueva América.

Zucker, A. (2005). Consorcio de Evaluación de Computación Uno a Uno: informe de política. Recuperado en febrero de 2009, de http:// idbdocs.iadb.org/wsdocs/getdocument. aspx? docnum $=895170$

Zuluaga, O. L., et. al. (2003). Pedagogía y Epistemología. Bogotá: Editorial Magisterio.

Vidart, D. (1997). Filosofía Ambiental. El ambiente como sistema. Bogotá: Editorial Nueva América. 